\title{
Effect of $\alpha$-mangostin on Enhanced Transdermal Bioavailability of Gartanin via Efflux Transporters
}

\author{
Rukthong $\mathbf{P}^{1}$, Sereesongsang $\mathbf{N}^{1}$, Kulsirirat $\mathrm{T}^{1}$, Nimprayoon $\mathbf{B}^{1}$ and Sathirakul $\mathbf{K}^{1,2^{*}}$ \\ ${ }^{1}$ Faculty of Pharmacy, Mahidol University, Bangkok, Thailand \\ ${ }^{2}$ Drug Discovery and Development Center, Thammasart University, Pathum Thani, Thailand
}

\begin{abstract}
Mangosteen (Garcinia mangostana $L$ ) is a tropical evergreen tree growing in Southeast Asia and has been used as traditional medicine treatment for skin wounds and infection. The pericarp crude extract can be isolated to fifty xanthone compounds, $\alpha-, \beta$ and $y$-mangostins, gartanin etc. This study aimed to characterize and compare the transdermal transport of $\alpha$-mangostin and gartanin when used alone and co-administered in human epidermal keratinocyte cells, neonatal (HEKn cells). The concentrations of the compounds were determined of by LC-MS/MS. In the absorptive direction, gartanin could not be detected during the entire 8 hour. Moreover, apparent permeability coefficient in secretory direction $\left(P_{\text {app }}, B-A\right)$ was significantly higher than that of absorptive direction $\left(P_{\text {app }}, A-B\right)$ but not found in $\alpha$-mangostin. The results showed that after incubating the HEKn cells with rotenone, $P_{\text {app }}, A-B$ of gartanin was significantly increased. In contrast, $P_{\text {app }}, A-B$ of $\alpha$-mangostin with and without rotenone was unchanged. For the mixture of gartanin and $\alpha$-mangostin, $\alpha$-mangostin had the similar inhibitory effect to the uptake and secretion of gartanin to the effect of rotenone. These indicated that the effect of efflux transporter of gartanin could be inhibited by $\alpha$-mangostin and the permeability of gartanin in absorptive direction was achieved with co-administration of a-mangostin at high concentration. It is postulated that alpha-mangostin may act as a natural enhancer to improve the bioavailability of gartanin. The synergistic effect of the co-existing of the compounds in the natural extract may be important for therapy.
\end{abstract}

Keywords: Bioavailability; Garcinia mangostana L.; a-Mangostin; Gartanin; Transdermal permeability; Active transport; Synergistic effect

\section{Introduction}

Mangosteen (Garcinia mangostana L.) is a popular refreshing juicy fruit in the summer. Moreover, their rinds have been used as a traditional medicine in Thailand for the treatment of trauma, diarrhea and skin infections [1]. The xanthones, $\alpha$-and $\gamma$-mangostins are major bioactive compounds found in the hulls of the mangosteen [2]. The biological activities of xanthones include a competitive antagonism of the histamine H1 receptor, antibacterial activity against Helicobacter pylori, anti-inflammatory activities $[3,4]$, inhibition of oxidative damage by human Low-density Lipoproteins (LDL) [5], antimicrobial activity against methicillin-resistant Staphylococcus aureus [6] and weak antioxidant activity [7]. In addition, gartanin and $\alpha$-mangostin isolated from pericarp were demonstrated to possess potent antioxidant activity using the authentic ONOO- and SIN-1-derived ONOO-methods. The IC50 of gartanin for authentic ONOO- and SIN-1-derived ONOO- were 9.1 and $9.3 \mu \mathrm{M}$, respectively. The IC50 of $\alpha$-mangostin for authentic ONOO- and SIN-1-derived ONOO- were 12.2 and $<0.49 \mu \mathrm{M}$ [8].

Transdermal delivery has shown significant advantages over the oral route in terms of eliminating first-pass effect of the liver and preventing premature drug metabolism. Transdermal delivery also has advantages over hypodermic injections, which are painful, generate dangerous medical waste and pose the risk of disease transmission by needle re-use [9]. In addition, transdermal systems are non-invasive and can be selfadministered [10]. Nevertheless, transdermal permeation of stratum corneum, the outermost layer of skin, is generally considered as a major barrier against environmental stress $[11,12]$. The routes of penetration into the skin for exogenous chemical substances are appendageal and epidermal transport. However, there is increasing evidence that skin epithelial cells contain biochemical barrier systems. These include metabolic enzymes expressed in skin keratinocytes that convert various types of xenobiotic compounds into hydrophilic metabolites, which can then be pumped out of the body by the xenobiotic transporter [13-15]. Xenobiotic transporters have broad substrate specificity and are involved in uptake (influx) and secretion (efflux), thereby affecting cellular disposition of their substrates. Therefore, it would be reasonable that transporters expressed in skin function as either barrier or carrier systems that influence the skin absorption of drugs. Therefore, the aim of this study was to characterize and compare the transdermal transport of a-mangostin and gartanin from G. mangostana by using human epidermal keratinocyte cells, neonatal (HEKn cells). After postseeding in Transwell ${ }^{\circ}$ with $0.4 \mu \mathrm{m}$ pore polyester membrane insert, the uptake experiments were performed. Two xanthones were permeated through the HEKn cells in two directions, apical to basolateral and vice versa. In addition, the effects of two xanthones on the transdermal transport were investigated. The experiments were established not only for $\alpha$-mangostin and gartanin alone but also for co-administration of two xanthones. The samples were sampling at the designated time points and all samples were analyzed by using LC-MS/MS [16,17].

\section{Materials and Methods}

\section{Biological activity}

Primary cell culture: Human epidermal keratinocyte cells neonatal (HEKn cells) (Invitrogen ${ }^{\mathrm{TM}}$, Lot no.792375, Oregon, USA) were cultured in T-25 flask at $37^{\circ} \mathrm{C}$ in an atmosphere of $5 \% \mathrm{CO}_{2}$. The

*Corresponding author: Sathirakul K, Faculty of Pharmacy, Mahidol University, Bangkok, Thailand, Tel: 66-86-3208895; Email: pysks2001@yahoo.com.sg

Received May 22, 2017; Accepted June 19, 2017; Published June 29, 2017

Citation: Rukthong P, Sereesongsang N, Kulsirirat T, Nimprayoon B, Sathirakul K (2017) Effect of a-mangostin on Enhanced Transdermal Bioavailability of Gartanin via Efflux Transporters. J Bioequiv Availab 9: 455-462. doi: 10.4172/jbb.1000344

Copyright: ( $) 2017$ Rukthong P, et al. This is an open-access article distributed under the terms of the Creative Commons Attribution License, which permits unrestricted use, distribution, and reproduction in any medium, provided the original author and source are credited. 
adherent keratinocytes were cultured in low calcium $(0.09 \mathrm{mM})$, serumfree, Epilife medium, and supplemented with concentrated (100X) solution of Human Keratinocyte Growth Supplement (HKGS) at $1 \%$ $\mathrm{v} / \mathrm{v}$ concentration. The medium was replaced regularly three times a week until the flask reaches $90 \%$ confluence. The cells were moved from the flasks by incubating the monolayers with $0.5 \%$ trypsin for 2-3 $\min$ at $37^{\circ} \mathrm{C}$. The cells were collected into centrifuge tubes, and then centrifuged at 1,000 rpm for $4 \mathrm{~min}$ and were re-suspended in Epilife medium. Cells used for this study were in third and fourth passage in late sub-confluency.

Culturing of HEKn cells on permeable supports: Cells were seeded at a density of $1 \times 10^{5}$ cells $/ \mathrm{cm}^{2}$ on permeable supports (Transwell Costar, $0.4 \mu \mathrm{m}$ pore size). The inserts were fed with complete media every other day until they were used in the experiments (3-5 days after seeding). The last medium replacement was occurred 24 hours before the transport studies started. Before the experiments, the cells were washed two times with $1 \mathrm{ml}$ of D-PBS. Then, xanthones dissolved in DMSO were added to the cell cultures with a final DMSO concentration of $0.1 \% \mathrm{v} / \mathrm{v}$, which had no significant effect on the growth and differentiation of HEKn. DMSO $(0.1 \%)$ treatment was used as control. Also, mannitol which permeates through the aqueous domain was used as a marker compound. The cells were incubated at $37^{\circ} \mathrm{C}$ for the designated time.

Viable cell number by trypan blue exclusion assay: HEKn cells were plated $\left(0.5 \times 10^{5} \mathrm{cells} / \mathrm{cm}^{2}\right.$ in 24 -well plates $)$. Twenty-four hours after plating, cells were treated with complete medium (blank), with the solvent of the compounds (0.1\% DMSO, control), and with test compounds. Viable cell numbers were assessed following 1, 4 and $8 \mathrm{~h}$ of incubation with the compounds using the trypan blue exclusion assay.

\section{Drug transport studies}

Passive drug transport: The drug solutions were prepared by dissolving test compounds: gartanin and $\alpha$-mangostin in media and filtered through a $0.22 \mu \mathrm{m}$ filter fitted to a plastic syringe into a test tube. Drug solutions $(0.8 \mathrm{ml})$ were added to the apical chambers and media $(1.2 \mathrm{ml})$ were added to the basolateral chambers. The media (200-400 $\mu \mathrm{l})$ were sampled from the basolateral chambers after $30 \mathrm{~min}, 1,2,3,4$, 6 and 8 h. Each sampling was replaced with completed fresh media and the concentrations of the samples were determined by LC-MS/MS.

Active drug transport: All the steps were performed as in passive drug transport but added the drug solutions to the basolateral chamber. The samples (200-350 $\mu \mathrm{l})$ were withdrawn from the apical receiving chamber and the fresh media were replaced in the apical chamber at regular time intervals. The permeability coefficient of the drug across the cell monolayers was calculated and the concentrations of the samples were determined by LC-MS/MS

\section{Liquid Chromatography-Mass Spectrometry (LC-MS)}

The LC-MS/MS system was consisted of a Q-Trap 5500 triple quadrupole/ion trap mass spectrometer (ABSCIEXTM, USA) equipped with a Turbo Spray ion source operated at $350^{\circ} \mathrm{C}$. The system included an Agilent 1200 series HPLC pump, degasser, auto sampler and column heater (Agilent, USA). The quantitative analysis of gartanin, $\alpha$-mangostin and mannitol by LC-MS/MS was validated according to accordance with USA Food and Drug Administration (FDA) bioanalytical method validation guidelines [18]. A method validation was carried out for specificity, sensitivity, linearity, recovery, precision, accuracy, and stability. The results showed that the method was accurate and precise for the quantification of gartanin, $\alpha$-mangostin and mannitol samples available in permeability studies.
Marker compound (mannitol): The HPLC separation of mannitol was performed using a $50 \times 2.1 \mathrm{~mm}$, Luna $5 \mu \mathrm{m} \mathrm{NH} 2100 \AA$ column (Agilent, USA) operated at a flow rate of $1 \mathrm{ml} / \mathrm{min}$. Water (A) and (B) acetonitrile was used as a mobile phase with the following gradient: $30 \%$ B for $0.75 \mathrm{~min}, 30$ to $90 \%$ B from 0.75 to $7 \mathrm{~min}$, and finally $30 \%$ B isocratic from 7 to $12 \mathrm{~min}$. An injection volume of $5 \mu \mathrm{l}$ was used for all analyses.

Xanthone samples ( $\boldsymbol{\alpha}$-mangostin and gartanin): HPLC separations of the xanthones from the samples were performed on a C18 reverse column (RP-C18, $5 \mu \mathrm{m}, 2.0 \times 150 \mathrm{~mm}$ column) (Mightysil, Tokyo, Japan). A mobile phase system of (A) water with $0.3 \%$ formic acid and (B) acetonitrile is used with the following gradient: $60 \%$ B for $5 \mathrm{~min}$, 60 to $95 \%$ B from 0.5 to $6 \mathrm{~min}, 95$ to $60 \%$ B from 6 to 8 min and finally $60 \% \mathrm{~B}$ isocratic from 8 to $12 \mathrm{~min}$ (flow rate $=1 \mathrm{ml} / \mathrm{min}$ ). An injection volume of $5 \mu \mathrm{l}$ was used for all analyses. The concentration ratio of both compounds in crude extract from pericarp was also determined.

\section{Calculation of permeability and apparent permeability coefficient}

Permeability: The permeability or flux $\left(\mathrm{J}, \mathrm{ng} / \mathrm{cm}^{2} / \mathrm{h}\right)$ was calculated as in Equation 1:

$$
J=\frac{d M}{d t} \times \frac{1}{A}
$$

Where $\mathrm{J}$ is the permeability or flux $\left(\mathrm{ng} / \mathrm{cm}^{2} / \mathrm{h}\right)$;

$\mathrm{dM} / \mathrm{dt}$ is the amount of drug transported across the membrane $(\Delta \mathrm{M})$ during the time $(\Delta \mathrm{t})$;

A is the cross-sectional area of the membrane of the Transwell $\left(1.2 \mathrm{~cm}^{2}\right)$.

Apparent permeability coefficient: The apparent permeability coefficient, $\mathrm{P}_{\text {app }}(\mathrm{nm} / \mathrm{s})$ was calculated as in Equation 2:

$$
P_{a p p}=\frac{d M}{d t} \times \frac{1}{A x C}
$$

where $\mathrm{P}_{\text {app }}$ is the apparent permeability coefficient $(\mathrm{nm} / \mathrm{s})$;

$\mathrm{dM} / \mathrm{dt}$ is the amount of drug transported across the membrane $(\Delta \mathrm{M})$ during the time $(\Delta \mathrm{t})$;

A is the cross-sectional area of the membrane of the Transwell $\left(1.2 \mathrm{~cm}^{2}\right)$;

$\mathrm{C}$ is the initial concentration of the compound added into the apical compartment ( $\mathrm{ng} / \mathrm{ml})$.

\section{ATP-dependent transdermal permeation study}

The transport via active transporter utilizes ATP for transporting their substrates in the opposite direction to the chemical gradient. The ATP-depressor, rotenone, has been used for verifying the effect of efflux transporter to the permeation of compounds across HEKn cells.

\section{Results}

\section{Quantitative analysis of gartanin, $\alpha$-mangostin and mannitol by LC-MS/MS}

The Q1 and Q3 quadrupoles were tuned for unit mass resolution. Multiple Reaction Monitoring (MRM) methods were used to confirm the identity of the xanthone analogs. MRM transitions for the xanthone compounds were 379.2/285.0, 411.2/355.1 and 181.0/89.0 in gartanin, 
Citation: Rukthong P, Sereesongsang N, Kulsirirat T, Nimprayoon B, Sathirakul K (2017) Effect of a-mangostin on Enhanced Transdermal Bioavailability of Gartanin via Efflux Transporters. J Bioequiv Availab 9: 455-462. doi: 10.4172/jbb. 1000344

a-mangostin and mannitol, respectively. The conditions used for LC-MS/MS quantitative analyzed of the retention time of gartanin, $\alpha$-mangostin and mannitol were $6.80,6.93$ and $0.89 \mathrm{~min}$. The analytical system was proved to validate for system suitability in terms of linearity, precision, specificity, accuracy and recovery. The concentration ratio of a-mangostin and gartanin in crude extract from pericarp was about 20:1 (n=6).

\section{ATP-dependent transdermal permeation of gartanin, a-mangostin and mannitol}

Mannitol: The result showed that after incubating the HEKn cells with $30 \mu \mathrm{M}$ rotenone for $30 \mathrm{~min}$, no effect on the permeation of mannitol was observed in the presence of rotenone in $\mathrm{P}_{\text {app }}, \mathrm{A}-\mathrm{B}$ and $\mathrm{P}_{\text {app' }}$, B-A (Figure 1). Because mannitol was permeated via passive transport pathway, so that ATP did not necessary for their transporting.

Gartanin: $\mathrm{P}_{\text {app }}$, A-B of gartanin when incubated the cells with 30 $\mu \mathrm{M}$ rotenone with was significantly increased whereas the $\mathrm{P}_{\text {app }}, \mathrm{B}-\mathrm{A}$ was significantly decrease $(\mathrm{p}<0.05)$ (Figure 2$) . \mathrm{P}_{\text {app }}, \mathrm{A}-\mathrm{B}$ was increased because the ability of efflux transporter for gartanin was decreased. The gartanin could permeate into the cells and then across the monolayer in the basolateral side of the cells. In contrast, the $\mathrm{P}_{\text {app }}$, B-A was decreased because there was no effect of the efflux transport on the permeation of the compound across the monolayer in the apical side. In this case, the permeation was observed only via

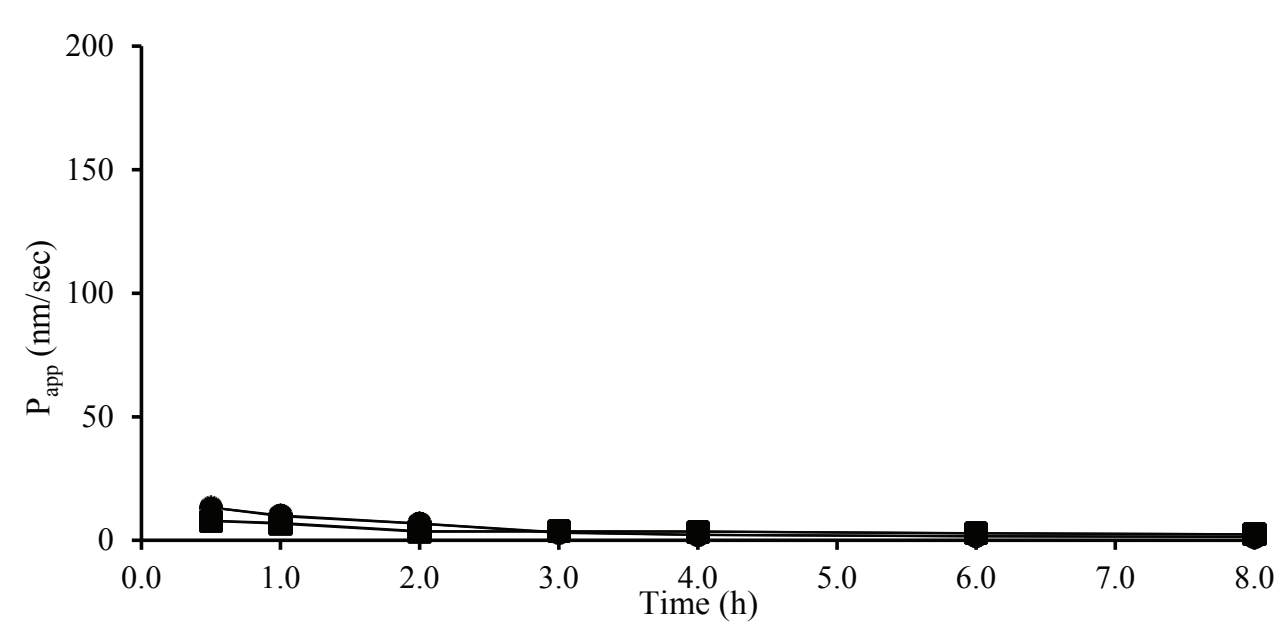

Figure 1: Inhibitory effect of rotenone (ATP-depressor) on the Papp of mannitol (137 $\mu \mathrm{M})$. The inhibitor effect of rotenone on the Papp of mannitol in both absorptive and secretory direction ( - and - , respectively) were compared with the Papp of mannitol when absence of rotenone in both absorptive and secretory direction ( -0 and $-\square$-, respectively). Each data represented the mean \pm SD of three separated replicate experiments.

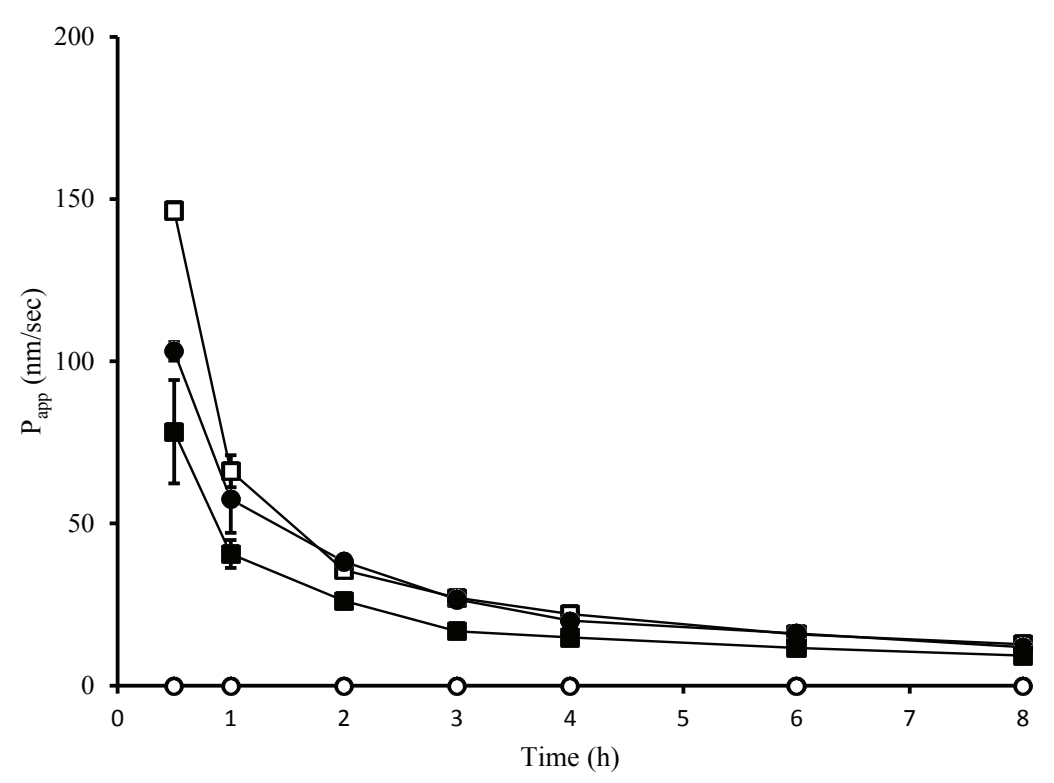

Figure 2: Inhibitory effect of rotenone (ATP-depressor) on the Papp of gartanin ( $9 \mu \mathrm{M})$. The inhibitor effect of rotenone on the Papp of gartanin in both absorptive and secretory direction (-and-arespectively) were compared with the Papp of gartanin when absence of rotenone in both absorptive and secretory direction $(-\circ$ and $-\square-$, respectively). Each data represented the mean \pm SD of three separated replicate experiments. 
Citation: Rukthong P, Sereesongsang N, Kulsirirat T, Nimprayoon B, Sathirakul K (2017) Effect of a-mangostin on Enhanced Transdermal Bioavailability of Gartanin via Efflux Transporters. J Bioequiv Availab 9: 455-462. doi: 10.4172/jbb. 1000344

passive transport pathway (Figure 3A and 3B). This finding indicated that the permeation of gartanin was involved in the ATP-dependent transport system.

a-Mangostin: There were no significant changes among the $\mathrm{P}_{\text {app }}$ A-B and $\mathrm{P}_{\text {app, }}, \mathrm{B}-\mathrm{A}$ of $\alpha$-mangostin with and without rotenone $(30 \mu \mathrm{M})$ (Figure 4). This indicated that the transport of $\alpha$-mangostin was not involved in the ATP-dependent transport system.

\section{Effect of $\alpha$-mangostin on the uptake of gartanin}

To perform the effect of $\alpha$-mangostin on the uptake of gartanin, the co-administration of these two compounds were determined. The uptake of $9 \mu \mathrm{M}$ gartanin was significantly increased when coadministration with $12 \mu \mathrm{M}$ a-mangostin ( $\mathrm{P}_{\text {app }}, \mathrm{A}-\mathrm{B}$ was increased). In contrast, the secretion of gartanin was significantly decreased when co-administration with a-mangostin ( $\mathrm{P}_{\text {app }}$, B-A was decreased) (Figure 5). This is owing to the inhibition effect of $\alpha$-mangostin on the efflux transporter of gartanin. There were two possible pathways to inhibit the efflux transporter. First, a-mangostin might perform as a pure inhibitor by only preventing gartanin to bind with their transporter. Second, a-mangostin might function as a competitive substrate of the transporter. Nevertheless, the previous studies revealed that rotenone did not affect the permeation of $\alpha$-mangostin. As a result, it is possible that $\alpha$-mangostin inhibits the efflux transporter as the pure inhibitor. However, when compared the $\mathrm{P}_{\text {app }}$, $\mathrm{A}-\mathrm{B}$ and $\mathrm{P}_{\text {app }}, \mathrm{B}-\mathrm{A}$ of gartanin and $\alpha$-mangostin mixture with $\mathrm{P}_{\text {app }}, \mathrm{A}-\mathrm{B}$ and $\mathrm{P}_{\text {app }}, \mathrm{B}-\mathrm{A}$ of gartanin and rotenone mixture, $\alpha$-mangostin had similar inhibition effect on the permeation of gartanin to rotenone (Figure 5). Alphamangostin might also inhibit the production of ATP like rotenone which might be possible pathways to explain the function of efflux transporter on gartanin (Figure 6).

\section{Effect of $\alpha$-mangostin concentration on the uptake of gartanin}

To support the possible involvement of $\alpha$-mangostin to the uptake of gartanin, gartanin was mixed with the various initial concentrations of $\alpha$-mangostin and the permeation in bidirectional transport assay was examined. The uptake was established in both absorptive (Figure 7A and Table 1) and secretory directions (Figure $7 \mathrm{~B}$ and Table 2) at 4 different initial concentrations $(0.375,0.75,3$ and $12 \mu \mathrm{M})$ of $\alpha$-mangostin with constant concentration of gartanin $(9 \mu \mathrm{M})$. In the first $30 \mathrm{~min}$ of the experiments, at initial concentration of $0.75,3$ and $12 \mu \mathrm{M} \alpha$-mangostin, $\mathrm{P}_{\text {app }}$, A-B of gartanin were still unchanged and they were significantly higher than that $\mathrm{P}_{\text {app }}, \mathrm{A}-\mathrm{B}$ at initial concentration of $0.375 \alpha$-mangostin. Likewise, $\mathrm{P}_{\text {app }}, \mathrm{B}-\mathrm{A}$ of gartanin, at $0.75,3$ and $12 \mu \mathrm{M}$, $\alpha$-mangostin were unchanged but they were significantly higher than that of $\mathrm{P}_{\text {app }}, \mathrm{A}-\mathrm{B}$ of gartanin at initial concentration of $0.375 \mu \mathrm{M}$ of $\alpha$-mangostin. These could be led by the interaction of the lead compound (gartanin) and

A
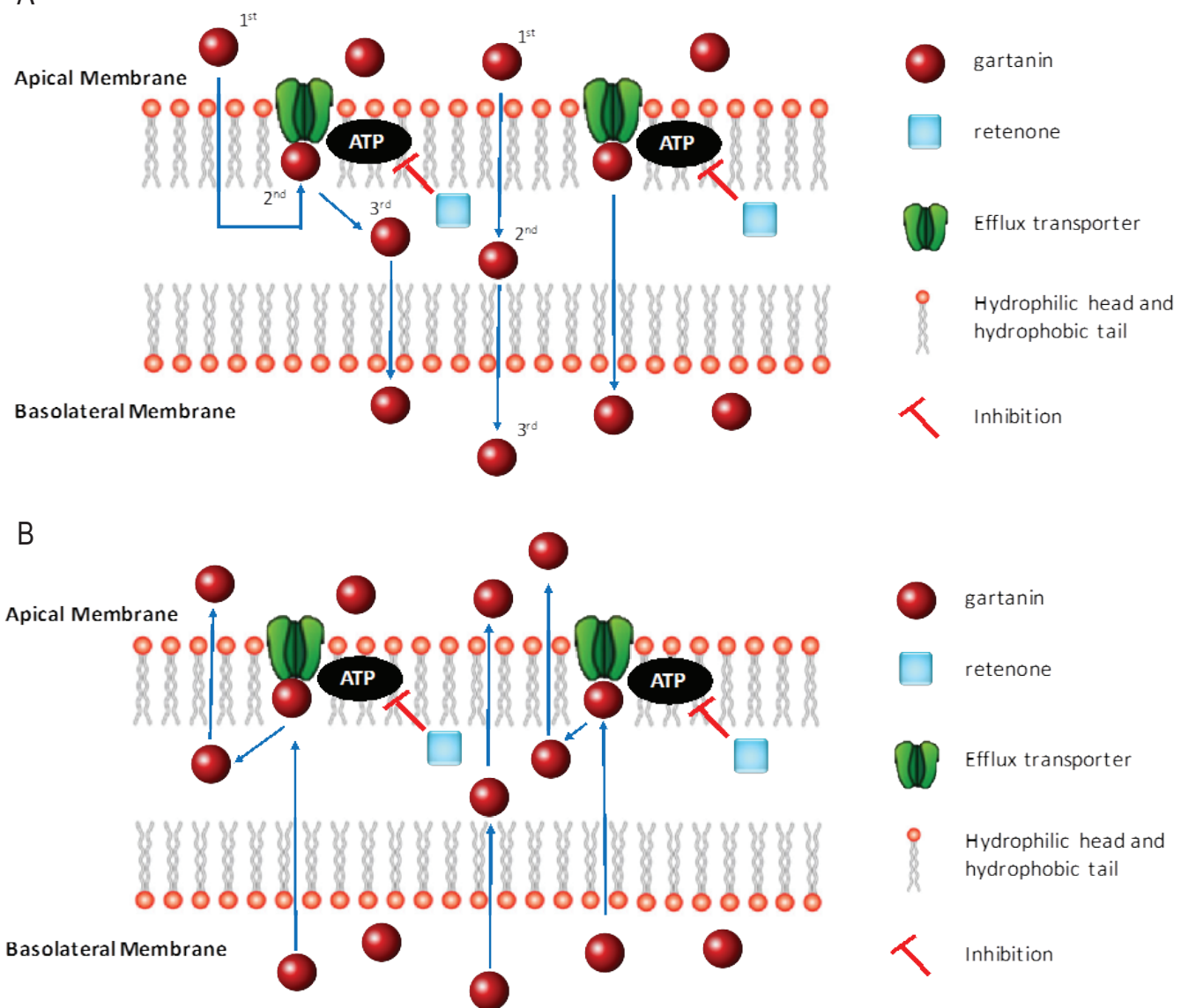

Figure 3: (A) The effect of rotenone to the permeation of gartanin in absorptive direction. (B) The effect of rotenone to the permeation of gartanin in secretory direction. 
Citation: Rukthong P, Sereesongsang N, Kulsirirat T, Nimprayoon B, Sathirakul K (2017) Effect of a-mangostin on Enhanced Transdermal Bioavailability of Gartanin via Efflux Transporters. J Bioequiv Availab 9: 455-462. doi: 10.4172/jbb.1000344

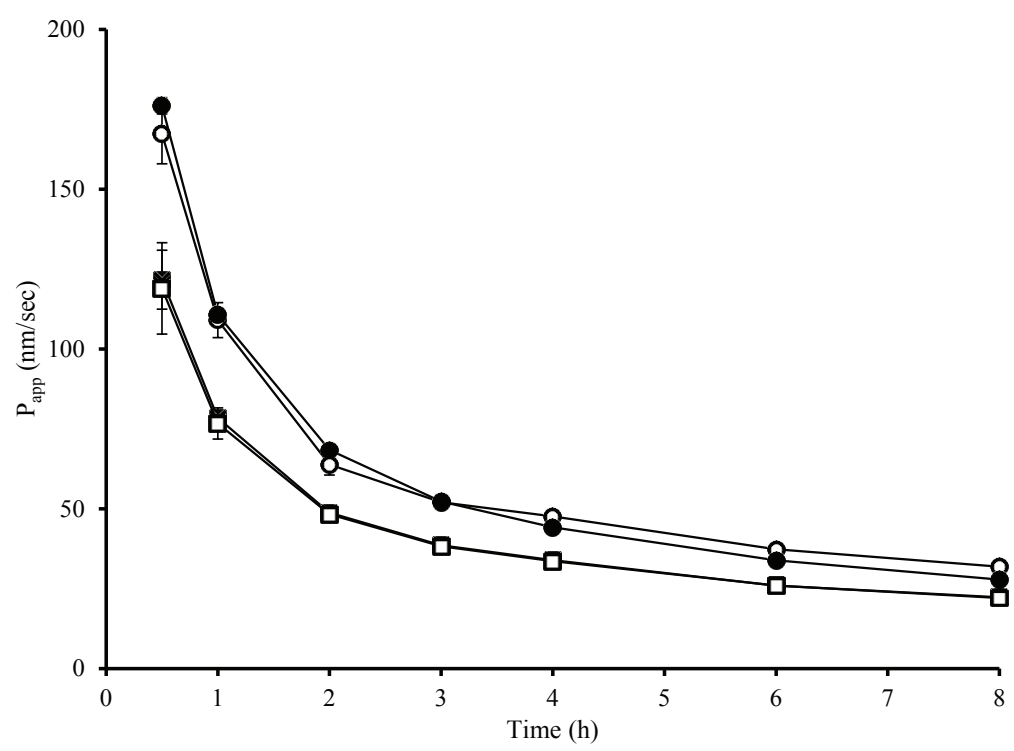

Figure 4: Inhibitory effect of rotenone (ATP-depressor) on the Papp of $\alpha$-mangostin $(12 \mu \mathrm{M})$. The inhibitor effect of rotenone on the Papp of $\alpha-m a n g o s t i n$ in both absorptive and secretory direction(- and- - respectively) were compared with the Papp of $\alpha$-mangostin when absence of rotenone in both absorptive and secretory direction ( $\left(\circ-\right.$ and $\_$- respectively). Each data represented the mean \pm SD of separated replicate experiments.

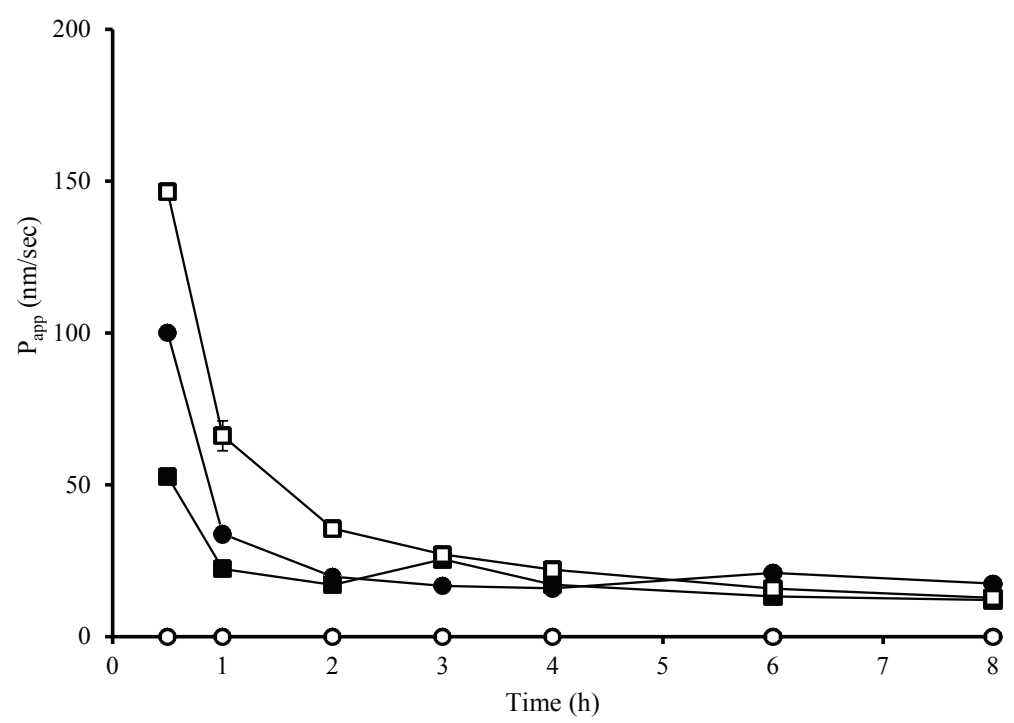

Figure 5: Effect of $\alpha$-mangostin $(12 \mu \mathrm{M})$ on the uptake of gartanin $(9 \mu \mathrm{M})$. The uptake was examined in absorptive and secretory direction (-and-a-, respectively) and compared with the Papp of gartanin when absence of $\alpha$-mangostin in both absorptive and secretory direction ( -0 - and $-\square-$, respectively). Each data represented the mean \pm SD of three separated replicate experiments.

candidate compound ( $\alpha$-mangostin) with the transporters. At initial low concentration (0.375) of a-mangostin, some effective transports were observed which could pump out their substrates from basolateral to apical side. Therefore, the $\mathrm{P}_{\text {app }}$, A-B of 0.375 of $\alpha$-mangostin were significant lower and $\mathrm{P}_{\text {app }}, \mathrm{B}-\mathrm{A}$ were higher than others initial concentrations. Meanwhile, at initial high concentrations (0.73, 3 and 12) of $\alpha$-mangostin, the transporter had been completely inhibited by the inhibitor and the high $\mathrm{P}_{\text {app }}, \mathrm{A}-\mathrm{B}$ of gartanin was observed. Nevertheless, at the end point of the experiments, $\mathrm{P}_{\text {app }}, \mathrm{A}-\mathrm{B}$ and $\mathrm{P}_{\text {app' }}$, $\mathrm{B}-\mathrm{A}$ of gartanin in all four concentrations were significantly changed.

\section{Efflux ratios of gartanin and $\alpha$-mangostin}

Gartanin: Effect of $\alpha$-mangostin concentrations $(0.375,0.75,3$ and $12 \mu \mathrm{M})$ and rotenone $(30 \mu \mathrm{M})$ were examined on the gartanin efflux ratios, $\mathrm{EfR}$, (ration of $\mathrm{P}_{\text {app }}, \mathrm{B}-\mathrm{A} / \mathrm{P}_{\text {app }}, \mathrm{A}-\mathrm{B}$ ) in bidirectional assay. Generally, the EfR was termed as passive transport when calculated $\mathrm{EfR}<2$. While, the substrate transport via the efflux transporter at the apical membrane was expressed with the EfR $>2$. As shown in Table 3, a-mangostin was involved with the efflux ratios of gartanin when compared with the efflux ratios of gartanin without $\alpha$-mangostin. At the initial concentrations of $0.375,0.75,3$ and $12 \mu \mathrm{M} \alpha$-mangostin, 


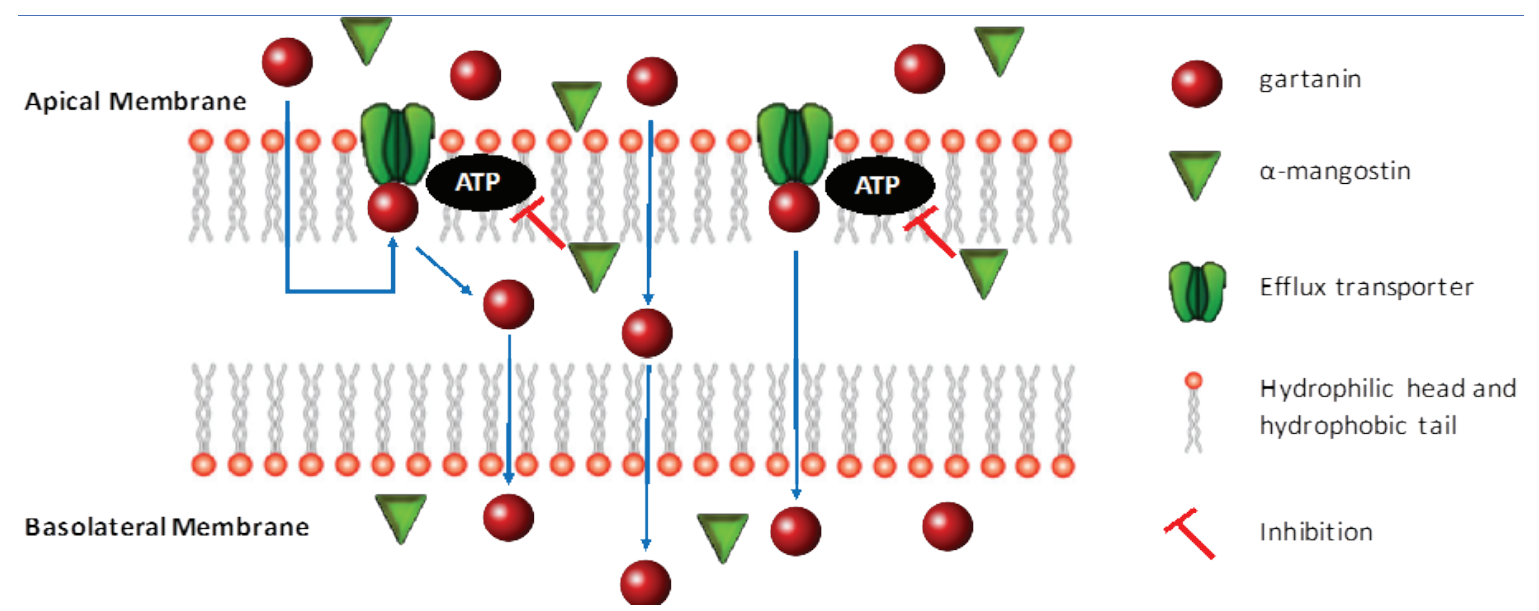

Figure 6: The possible pathway of $\alpha$-mangostin to inhibit the efflux transporters for gartanin (as the ATP-depressor).
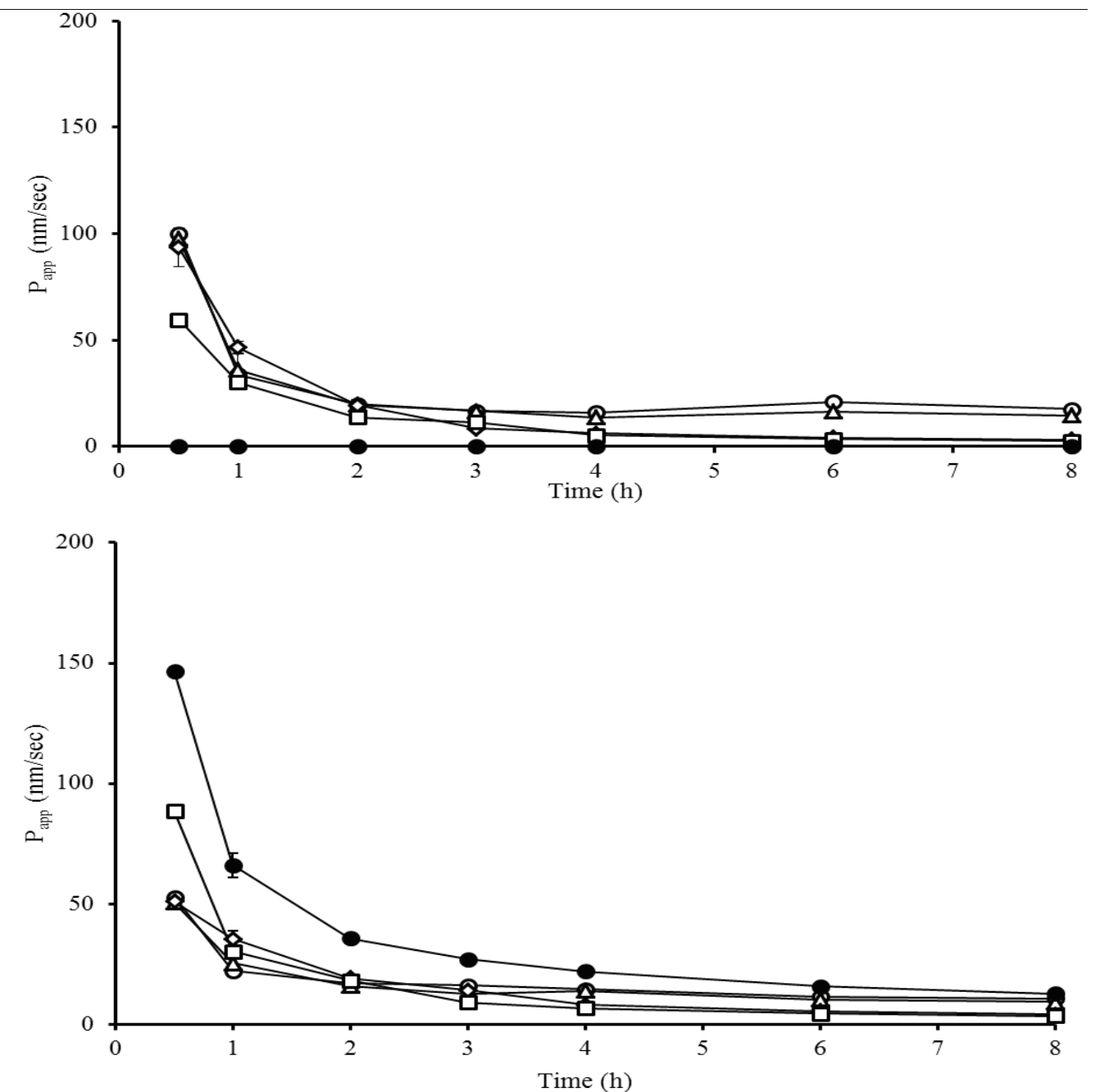

Figure 7: Effect of $\alpha$-mangostin concentrations $(0,0.375,0.75,3$ and $12 \mu \mathrm{M})$ on the uptake of gartanin in bidirectional study. In panel A and B, the Papp, A-B and Papp, B-A of gartanin when co-administration with various concentrations of $\alpha$-mangostin were shown. $(\bullet, 0 \mu \mathrm{M},-\square-0.375 \mu \mathrm{M}, \diamond 0.75 \mu \mathrm{M} ;-\Delta-3 \mu \mathrm{M},-\Omega \mu \mathrm{M}) \mathrm{Each}$ data represent the mean \pm SD of three separated replicate experiments. 
Citation: Rukthong P, Sereesongsang N, Kulsirirat T, Nimprayoon B, Sathirakul K (2017) Effect of a-mangostin on Enhanced Transdermal Bioavailability of Gartanin via Efflux Transporters. J Bioequiv Availab 9: 455-462. doi: 10.4172/jbb.1000344

\begin{tabular}{|c|c|c|c|c|c|}
\hline \multirow{2}{*}{$\begin{array}{c}\text { Time (h) } \\
0.0\end{array}$} & \multicolumn{5}{|c|}{$\begin{array}{l}\text { Papp, A-B of gartanin }(\mathrm{nm} / \mathrm{s}) \pm \mathrm{SD} \\
\alpha-\text { mangostin concentration }(\mu \mathrm{M})\end{array}$} \\
\hline & 0.375 & 0.750 & 3.000 & 12.000 & controlled \\
\hline 0.5 & $59.598 \pm 0.835^{\mathrm{a}}$ & $93.583 \pm 9.002^{a, b}$ & $97.566 \pm 3.009^{a, b}$ & $100.100 \pm 0.933^{a, b}$ & $0.000 \pm 0.000$ \\
\hline 1 & $30.113 \pm 0.387^{a}$ & $46.606 \pm 2.891^{a, b}$ & $35.965 \pm 7.661^{a-c}$ & $33.793 \pm 0.779^{a-c}$ & $0.000 \pm 0.000$ \\
\hline 2 & $13.695 \pm 0.123^{\mathrm{a}}$ & $19.406 \pm 0.833^{a, b}$ & $19.583 \pm 2.523^{a, b}$ & $19.743 \pm 1.343^{a, b}$ & $0.000 \pm 0.000$ \\
\hline 3 & $11.405 \pm 0.281^{a}$ & $8.341 \pm 0.506^{a, b}$ & $16.693 \pm 1.298^{a-c}$ & $16.686 \pm 0.890^{a-c}$ & $0.000 \pm 0.000$ \\
\hline 4 & $5.267 \pm 0.032^{\mathrm{a}}$ & $6.255 \pm 0.379^{a, b}$ & $13.735 \pm 0.337^{a-c}$ & $15.905 \pm 0.791^{a-d}$ & $0.000 \pm 0.000$ \\
\hline 6 & $3.511 \pm 0.021^{\mathrm{a}}$ & $4.170 \pm 0.253^{\mathrm{a}}$ & $16.452 \pm 0.751^{a-c}$ & $20.988 \pm 0.286^{a-d}$ & $0.000 \pm 0.000$ \\
\hline 8 & $2.633 \pm 0.016^{a}$ & $3.128 \pm 0.190^{a}$ & $14.623 \pm 1.544^{a-c}$ & $17.479 \pm 0.404^{a-d}$ & $0.000 \pm 0.000$ \\
\hline \multicolumn{6}{|c|}{$\begin{array}{l}\text { Significantly different to controlled (without } \alpha \text {-mangostin) } \\
\text { Significantly different to } 0.375 \mu \mathrm{M} \text { a-mangostin } \\
\text { Significantly different to } 0.750 \mu \mathrm{M} \text { a-mangostin } \\
\text { Significantly different to } 3.000 \mu \mathrm{M} \text { a-mangostin }\end{array}$} \\
\hline
\end{tabular}

Table 1: The effect of $\alpha$-mangostin in various concentrations on the apparent permeability of gartanin in absorptive direction $\left(\mathrm{P}_{\text {app }}\right.$, A-B). Each data represents the mean \pm $\mathrm{SD}$ of three experiments. ${ }^{\mathrm{d}}$ Significantly different to $3.000 \mu \mathrm{M} \alpha$-mangostin SD of three experiments.

\begin{tabular}{|c|c|c|c|c|c|}
\hline \multirow{2}{*}{$\begin{array}{c}\text { Time (h) } \\
0.0\end{array}$} & \multicolumn{5}{|c|}{$\begin{array}{l}P_{\text {app, }} \text { A-B of gartanin }(\mathrm{nm} / \mathrm{s}) \pm \mathrm{SD} \\
\alpha \text {-mangostin concentration }(\mu \mathrm{M})\end{array}$} \\
\hline & 0.375 & 0.750 & 3.000 & 12.000 & controlled \\
\hline 0.5 & $88.448 \pm 1.696^{a}$ & $50.999 \pm 2.85^{a, b}$ & $50.564 \pm 1.008^{a, b}$ & $52.792 \pm 1.093^{a, b}$ & $146.464 \pm 1.627$ \\
\hline 1 & $30.228 \pm 0.489^{a}$ & $35.497 \pm 3.504^{\mathrm{a}, \mathrm{c}}$ & $25.445 \pm 4.105^{\mathrm{a}}$ & $22.276 \pm 0.902^{a-c}$ & $66.126 \pm 4.936$ \\
\hline 2 & $18.146 \pm 0.191^{a}$ & $19.178 \pm 1.458^{\mathrm{a}, \mathrm{c}}$ & $15.903 \pm 2.340^{a}$ & $17.030 \pm 0.482^{a}$ & $35.626 \pm 0.560$ \\
\hline 3 & $9.058 \pm 0.107^{\mathrm{a}}$ & $14.128 \pm 0.758^{a, b}$ & $12.619 \pm 0.662^{a, b, d}$ & $16.197 \pm 1.185^{a-c}$ & $27.106 \pm 1.158$ \\
\hline 4 & $6.793 \pm 0.080^{\mathrm{a}}$ & $8.104 \pm 0.498^{a, c}$ & $14.044 \pm 0.824^{a, b}$ & $14.468 \pm 1.022^{a-c}$ & $22.045 \pm 0.875$ \\
\hline 6 & $4.529 \pm 0.053^{\mathrm{a}}$ & $5.403 \pm 0.332^{a-c}$ & $10.420 \pm 0.152^{a, b}$ & $11.487 \pm 0.806^{a-d}$ & $15.807 \pm 0.294$ \\
\hline 8 & $3.397 \pm 0.040^{\mathrm{a}}$ & $4.0523 \pm 0.249^{a-c}$ & $9.388 \pm 0.186^{a, b}$ & $10.748 \pm 0.115^{a-d}$ & $12.760 \pm 0.138$ \\
\hline \multicolumn{6}{|c|}{$\begin{array}{l}\text { Significantly different to controlled (without } \alpha \text {-mangostin) } \\
\text { Significantly different to } 0.375 \mu \mathrm{M} \text { a-mangostin } \\
\text { Significantly different to } 0.750 \mu \mathrm{M} \text {-mangostin } \\
\text { Significantly different to } 3.000 \mu \mathrm{M} \text { a-mangostin }\end{array}$} \\
\hline
\end{tabular}

Table 2: The effect of $\alpha$-mangostin in various concentrations on the apparent permeability of gartanin in secretory direction $\left(\mathrm{P}_{\text {app }}\right.$, B-A $)$. Each data represents the mean \pm $\mathrm{SD}$ of three experiments.

\begin{tabular}{|c|c|c|c|}
\hline \multirow{2}{*}{ Compounds } & $\begin{array}{c}\text { Concentration } \\
(\boldsymbol{\mu M})\end{array}$ & \multicolumn{2}{|c|}{ Gartanin $\mathbf{9} \boldsymbol{\mu M}$} \\
\cline { 3 - 4 } & 12 & $0.6992 \pm 0.0131$ & $1.4306 \pm 0.0270$ \\
\hline a-mangostin & 3 & $0.8123 \pm 0.0259$ & $1.2319 \pm 0.0385$ \\
\hline a-mangostin & 0.75 & $0.8720 \pm 0.0061$ & $1.1468 \pm 0.0081$ \\
\hline a-mangostin & 0.375 & $0.8711 \pm 0.0003$ & $1.1480 \pm 0.0004$ \\
\hline a-mangostin & 30 & $0.7940 \pm 0.0178$ & $1.2599 \pm 0.0279$ \\
\hline Rotenone & &
\end{tabular}

${ }^{a}$ Efflux ratio; 'Influx ratio; and ${ }^{\mathrm{c}} \mathrm{P}_{\text {app }}$ : A-B equal to zero along the experiment time

Table 3: Efflux and influx ratios of $\alpha$-mangostin and the effect of rotenone. The efflux and influx ratio was calculated by Papp, B-A/Papp, A-B and Papp, A-B/Papp $B-A$, respectively. Each data represents the mean \pm SD of three experiments.

efflux ratios were $1.2899 \pm 0.0209,1.3002 \pm 0.01318,0.6473 \pm 0.0759$ and $0.6151 \pm 0.0153$, respectively. These results indicated that coadministration of gartanin and $\alpha$-mangostin would be occurred via passive process. From the analysis, all of gartanin efflux ratios were not different to the efflux ratios of gartanin with rotenone. The finding suggested that $\alpha$-mangostin could inhibit the efflux transporter of gartanin.

a-Mangostin: To clarify the permeation of $\alpha$-mangostin, the efflux ratios of a-mangostin were also calculated (Table 4). According to the results, efflux ratios of four concentrations $\alpha$-mangostin $(0.375,0.75$, 3 and $12 \mu \mathrm{M})$ were all under $2(0.8711 \pm 0.0003,0.8720 \pm 0.0061$, $0.8123 \pm 0.0259$ and $0.6992 \pm 0.0131$, respectively). Thus, this could be confirmed that the permeation of a-mangostin did not transport via efflux transporter as gartanin. Moreover, $\alpha$-mangostin was prone

\begin{tabular}{|c|c|c|c|}
\multirow{2}{*}{ Compounds } & \multirow{2}{*}{ Concentration $(\boldsymbol{\mu M})$} & \multicolumn{2}{|c|}{ Gartanin $\mathbf{9} \boldsymbol{\mu M}$} \\
\cline { 2 - 4 } & & $\mathbf{E f R}^{\mathrm{a}} \pm \mathbf{S D}$ & IfR $^{\mathrm{b}} \pm \mathbf{S D}$ \\
\hline a-mangostin & 12 & $0.6151 \pm 0.0153$ & $1.6264 \pm 0.0399$ \\
\hline a-mangostin & 3 & $0.6473 \pm 0.0759$ & $1.5601 \pm 0.1958$ \\
\hline a-mangostin & 0.75 & $1.3002 \pm 0.1318$ & $0.7747 \pm 0.0833$ \\
\hline a-mangostin & 0.375 & $1.2899 \pm 0.0209$ & $0.7754 \pm 0.0127$ \\
\hline Rotenone & 30 & $0.7845 \pm 0.0567$ & $1.2791 \pm 0.0933$ \\
\hline Gartanin & 9 & na $^{\mathrm{c}}$ & $0.0000 \pm 0.0000$ \\
\hline
\end{tabular}

Table 4: Effect of $\alpha$-mangostin concentrations and rotenone on the gartanin efflux and influx ratio. The efflux and influx ratio was calculated by $P_{a p p}, B-A / P_{a p p}, A-B$ and $P_{a p p}, A-B / P_{a p p}, B-A$, respectively. Each data represents the mean $\pm S D$ of three experiments.

to the absorptive direction than secretory direction. To verify this information, the Influx Ratios (IfR) were clarified. The IfR of four concentrations $\alpha$-mangostin $(0.375,0.753$ and $12 \mu \mathrm{M})$ were all under $2(1.1480 \pm 0.0004,1.1468 \pm 0.0081,1.2319 \pm 0.0385$ and $1.4306 \pm$ 0.0270 , respectively). These assured that transport of $\alpha$-mangostin from basolateral to apical side was limited as compared to the transport when a-mangostin was added to the apical side.

\section{Discussion}

In the present study, the possible involvement of the active transport system of two compounds (gartanin and $\alpha$-mangostin) was demonstrated. The bi-directional studies were conducted across the HEKn cells. Mannitol was used as the marker compound to compare 
Citation: Rukthong P, Sereesongsang N, Kulsirirat T, Nimprayoon B, Sathirakul K (2017) Effect of a-mangostin on Enhanced Transdermal Bioavailability of Gartanin via Efflux Transporters. J Bioequiv Availab 9: 455-462. doi: 10.4172/jbb.1000344

and clarify the permeation of the test compounds. To confirm the result, ATP-depressor, rotenone was used to inhibit the activity of the transporter.

Quantitative determination of the compounds by using LC-MS/ MS system revealed that gartanin could not transport across the HEKn cells in the absorptive direction along the experiment time. In contrast, the $\mathrm{P}_{\text {app }}$, B-A of gartanin was over 5-fold higher than that $\mathrm{P}_{\text {app }}, \mathrm{B}$-A of mannitol. This finding showed the involvement of the active transport of gartanin with the specific efflux transporter in the permeation. The ATP is essential for active transport process, the increasing of $\mathrm{P}_{\text {app' }}$, A-B of gartanin when incubated the cells with rotenone could confirm the involvement of the efflux transporter of gartanin. Likewise, the absorption of gartanin could be altered when co-administration with a-mangostin.

Then, four different initial concentrations of gartanin, $\alpha$-mangostin were investigated on the uptake studies. The $\mathrm{P}_{\text {app }}, \mathrm{A}-\mathrm{B}$ of $0.375 \mu \mathrm{M}$ initial concentration was significantly lower than others initial concentrations and $\mathrm{P}_{\text {app }}, \mathrm{B}-\mathrm{A}$ was significantly higher than others initial concentrations. This finding was attributed to the fact that, at others initial high concentrations, the transporter had been completely inhibited by the inhibitor and the high $\mathrm{P}_{\text {app }}$, A-B of gartanin was observed. Therefore, the increasing of $\mathrm{P}_{\text {app }}$, $\mathrm{B}-\mathrm{A}$ has involved in a concentration-dependent mechanism. Moreover, the flux of gartanin was saturated at high concentration of $\alpha$-mangostin. The result suggested that gartanin was transport via carrier mediated transport system and could be reached the maximum velocity of the transported when co-administration with the high concentration of inhibitor ( $\alpha$-mangostin).

The synergistic effects of herbal drug were summarized currently [19]. Among all synergistic effects, the enhancement of the bioavailability of the compound in the herbal formulation is one of the major considerations. For our study, the use of single compound from the herbal extracts might be not provided the biological activities as expected owing to biological barrier systems of the skin. The coadministration could influence the permeation of the compounds which contributed to their effective activities. This is the first report to demonstrate the synergistic effect of natural available components in mangosteen pericarp. However, the similar observation was demonstrated in St. John's wort (Hypericum perforatum). The procyanidins in St. John's wort can increase the bioavailability of active compound in the herb; hypericin [20]. Thus, further studies need to be done in order to characterize the specific type of efflux transporters in each compound from the herbal plants and clarify the physiological roles of transporters in the skin that can lead to the identification of novel targets for transdermal delivery of drugs.

\section{Conclusion}

In this work, we have studied to the characterize and compare the transdermal transport of co-administration of a-mangostin and gartanin from Garcinia mangostana by using human epidermal keratinocyte cells, neonatal (HEKn cells) and determination of the compounds by using LC-MS/MS system. Alpha-Mangostin had the similar inhibition effect to the uptake and secretion of gartanin to the effect of rotenone. These indicated that the effect of efflux transporter of gartanin could be inhibited by $\alpha$-mangostin. It is possible pathway that alpha-mangostin may act as a co-effector to improve the bioavailability of gartanin. The LC-MS/MS methods used in this study were suitable for the quantitative analysis of gartanin, $\alpha$-mangostin and mannitol.

\section{Acknowledgments}

The authors gratefully acknowledge research grant from Commission on Higher Education (Staff Development Project), Thailand for financial support.

\section{References}

1. Chen LG, Yang LL, Wang CC (2008) Anti-inflammatory activity of mangostins from Garcinia mangostana. Food Chem Toxicol 46: 688-693.

2. Chairungsrilerd N, Takeuchi K, Ohizumi Y, Nozoe S, Ohta T (1996) Mangostanol, a prenylxanthone from Garcinia mangostana. Phytochem Rev 43: 1099-1102.

3. Chomnawang MT, Surassmo S, Nukoolkarn VS, Gritsanapan W (2007) Effect of Garcinia mangostana on inflammation caused by Propionibacterium acnes. Fitoterapia 78: 401-408.

4. Oprica C, Emtestam L, Lapins J, Borglund E, Nyberg F, et al. (2004) Antibioticresistant Propionibacterium acnes on the skin of patients with moderate to severe acne in Stockholm. Anaerobe 10: 155-164.

5. likubo K, Ishikawa Y, Ando N, Umezawa K, Nishiyama S (2002) The first direct synthesis of a-mangostin, a potent inhibitor of the acidic sphingomyelinase. Tetrahedron Lett 43: 291-293.

6. linuma M, Tosa H, Tanaka T, Asai F, Kobayashl Y, et al. (1996) Antibacterial Activity of Xanthones from Guttiferaeous Plants against Methicillin-resistant Staphylococcus aureus. J Pharm Pharmacol 48: 861-865.

7. Suvarnakuta P, Chaweerungrat C, Devahastin S (2011) Effects of drying methods on assay and antioxidant activity of xanthones in mangosteen rind Food Chem 125: 240-247.

8. Jung HA, Su BN, Keller WJ, Mehta RG, Kinghorn AD (2006) Antioxidant Xanthones from the Pericarp of Garcinia mangostana (Mangosteen). J Agric Food 54: 2077-2082

9. Miller MA, Pisani E (1999) The cost of unsafe injections. Bull World Health Organ 77: 808-811.

10. Prausnitz MR, Langer R (2008) Transdermal drug delivery. Nat Biotechnol 26 1261-1268.

11. Bouwstra JA, de Graaff A, Gooris GS, Nijsse J, Wiechers JW, et al. (2003) Water distribution and related morphology in human stratum corneum at different hydration levels. J Invest Dermatol 120: 750-758.

12. Wang TF, Kasting GB, Nitsche JM (2006) A multiphase microscopic diffusion model for stratum corneum permeability. I. formulation, solution, and illustrative results for representative compounds. J Pharm Sci Technol 95: 620-648.

13. Baron JM, Höller D, Schiffer R, Frankenberg S, Neis M, et al. (2001) Expression of multiple cytochrome p450 enzymes and multidrug resistance-associated transport proteins in human skin keratinocytes. J Invest Dermatol 116: 541-548.

14. Li Q, Kato Y, Sai Y, Imai T, Tsuji A (2005) Multidrug resistance-associated protein 1 functions as an efflux pump of xenobiotics in the skin. Pharm Res 22: 842-846.

15. Naczk M, Towsend M, Zadernowski R, Shahidi F (2011) Protein-binding and antioxidant potential of phenolics of mangosteen fruit (Garcinia mangostana). Food Chem 128: 292-298.

16. Skazik C, Wenzel J, Marquardt Y, Kim A, Merk HF, et al. (2011) P-Glycoprotein (ABCB1) expression in human skin is mainly restricted to dermal components. Exp Dermatol 20: 450-452.

17. Zhao Q, Dai C, Fan S, Lv J, Nie L (2016) Synergistic efficacy of salicylic acid with a penetration enhancer on human skin monitored by OCT and diffuse reflectance spectroscopy. Sci Rep 6.

18. US Food and Drug Administration (2001) Guidance for industry, bioanalytical methods validation. FDA.

19. Zhou X, Seto SW, Chang D, Kiat H, Razmovski-Naumovski V, et al. (2016) Synergistic: Effects of chinese herbal medicine: A comprehensive review of methodology and current research. Front Pharmacol 7: 1-16.

20. Nahrstedt A, Butterweck $V$ (2010) Lessons learned from herbal medicinal products: the example of St. John's wort. J Nat Prod 73: 1015-1021. 